\title{
Upregulation of phosphorylated cofilin 1 correlates with taxol resistance in human ovarian cancer in vitro and in vivo
}

\author{
MIN LI $^{1}$, JIE YIN $^{2}$, NING MAO $^{3}$ and LINGYA PAN ${ }^{2}$ \\ ${ }^{1}$ Department of Obstetrics and Gynecology, Beijing Hospital, Dongdan, Beijing 100730; \\ ${ }^{2}$ Department of Obstetrics and Gynecology, Peking Union Medical College Hospital, \\ Chinese Academy of Medical Science and Peking Union Medical College, Beijing 100730; \\ ${ }^{3}$ Department of Cell Biology, Institute of Basic Medical Science, Beijing 100850, P.R. China
}

Received July 10, 2012; Accepted August 13, 2012

DOI: $10.3892 / o r .2012 .2078$

\begin{abstract}
The acquisition of chemoresistance is a major therapeutic obstacle in the clinical treatment of ovarian cancer. Diagnosing chemoresistance in ovarian cancer patients at an early stage is necessary for prognosis, but at present significant proteins related to chemoresistance that may indicate and reverse chemoresistance in human ovarian cancer have not been discovered. In this study, we demonstrated that the protein, phosphorylated cofilin 1 (p-CFL1) correlates with taxol resistance in human ovarian cancer cells. The total proteins of two sensitive (SKOV3 and A2780) and three taxolresistant (SKOV3/TR2500, SKOV3/TR30 and A2780/TR) human ovarian cancer cell lines were isolated by 2-dimensional gel electrophoresis (2-DE). Twenty-two protein spots in all samples were revealed to be significantly different in spot intensity by statistical analysis, 16 of which were identified using matrix-assisted laser desorption ionization-time-of-flightmass spectrometry (MALDI-TOF-MS). Cofilin 1 (CFL1) was selected as a candidate which may play an important role in taxol resistance. Higher expression levels of p-CFL1 in taxolresistant cells were demonstrated. Furthermore, the levels of p-CFL1 in primary human ovarian cancer tissues were shown to be significantly higher in chemoresistant cases compared to those in chemosensitive ones. These findings suggest that p-CFL1 is upregulated in taxol-resistant ovarian cancer and this upregulation is a characteristic of taxol resistance both in vitro and in vivo. However, the mechanisms need to be further elucidated.
\end{abstract}

Correspondence to: Dr Lingya Pan, Department of Obstetrics and Gynecology, Peking Union Medical College Hospital, Chinese Academy of Medical Science and Peking Union Medical College (CAMS and PUMC), No. 1 Shuai Fu Yuan, Beijing 100730, P.R. China E-mail: lingyapan@hotmail.com

Key words: drug resistance, ovarian cancer, taxol, proteomics, phosphorylated cofilin 1

\section{Introduction}

Although ovarian cancer ranks 7 th among all cancers in women in terms of prevalence, almost $60-70 \%$ of those who have ovarian cancer eventually succumb to the disease (1). Ovarian cancer has a considerably higher mortality rate compared to breast cancer (approximately 20\% mortality rate), which has a much higher incidence $(1,2)$. In fact, ovarian cancer is the most lethal gynecologic malignancy (3). Failure to obtain better survival rates in ovarian cancer is caused by drug resistance. Over the past 3 decades, surgical tumor debulking, followed by chemotherapy is the standard treatment for advanced ovarian cancer. Although response rates and complete responses in advanced disease are $>80$ and $40-60 \%$, respectively, after a first-line treatment with carboplatin and paclitaxel, a majority of patients eventually relapse with a median progression-free survival of 18 months $(3,4)$. Prompt adjustment of the chemotherapy regimen based on early detection of tumor resistance to the drugs and discovering targets to reverse drug resistance may further improve the outcomes of this disease.

Lack of a detailed understanding of drug resistance mechanisms may delay, circumvent or prevent the development of drug resistance. Proper tools for studying drug resistance mechanisms are important but difficult to obtain in a clinical situation. A number of reported findings on paclitaxel resistance were achieved mainly by in vitro studies with cell lines that have acquired drug resistance. To date, possible drug resistance mechanisms reported include enhanced expression of P-glycoprotein, alterations in tubulin structure through gene mutations in the tubulin $\beta$ chain and changes in the ratio of tubulin isomers within the polymerized microtubule (5-8).

In view of the existence of post-translational protein processing and modification, inconsistencies were often identified between the expression levels of genes and proteins. The application of proteomic techniques may perform high throughput comparisons at the protein level and are ideally suitable in identifying differences in expressed proteins between chemosensitive and chemoresistant cells. In order to better understand chemoresistance in ovarian cancer, we established a protein screening in 2 human ovarian cancer cell lines and their taxol-resistant variants. Phosphorylated cofilin 1 (p-CFL1) 
was selected, validated in vivo and in vitro and a correlation between p-CFL1 and taxol resistance was confirmed.

\section{Materials and methods}

Cell culture, drugs and cytotoxicity assay. The human epithelial ovarian cancer cell line SKOV3, used in this study, was purchased from the Cell Culture Center, Institute of Basic Medical Science, Chinese Academy of Medical Sciences. Human epithelial ovarian cancer cell line A2780 and its taxolresistant subtype A2780/TR were kindly supplied by the Cancer Institute of the Guangxi Medical University. SKOV3/TR2500 and SKOV3/TR30, 2 taxol-resistant variants from SKOV3, were induced in our laboratory (9). For inducing SKOV3/ TR2500, SKOV3 was cultured in taxol $(6 \mathrm{mg} / \mathrm{ml}$; Bristol-Myers Squibb Co., USA) at $2.5 \mu \mathrm{M}$ for $1 \mathrm{~h}$, and the pulse was repeated 21 times for 18 months. For inducing SKOV3/TR30, cells underwent intermittent stimulation for $24 \mathrm{~h}$ in taxol at 10,20 and $30 \mathrm{nM}$ successively, repeating each concentration 10 times. The process lasted 12 months. All cell lines were maintained in Dulbecco's modified Eagle's medium/high glucose (DMEM/ HG) containing $10 \%$ fetal calf serum without or with taxol, respectively. Cells were cultured in a humidified incubator at $37^{\circ} \mathrm{C}$ with $5 \%$ carbon dioxide, $95 \%$ humidity and passaged when cultures were $70-80 \%$ confluent.

In vitro cytotoxicity was measured using a tetrazoliumbased semi-automated colorimetric (MTT) assay, as previously described $(10,11)$. The absorbance values were normalized assigning the value of the parent cell lines in the media without the drug to 1.0 and the value of the no-cell control to 0 . Experiments were performed in duplicate. The half maximal inhibitory concentration $\left(\mathrm{IC}_{50}\right)$ value (the concentration of drugs that produced a 50\% reduction in absorbance) and the relative resistance of treated cells were analyzed. The drug used was taxol $(6 \mathrm{mg} / \mathrm{ml})$.

To identify the level of CFL1 and p-CFL1 in chemosensitive and chemoresistant cell lines, cells were cultured in DMEM/HG without FBS for $48 \mathrm{~h}$ to delete the effects of the surrounding factors and were stimulated with $10 \mathrm{nM}$ taxol for $0,5,10,15,30$ and $60 \mathrm{~min}$, respectively. The cells were then collected immediately and total proteins were extracted.

Two-dimensional gel electrophoresis (2-DE) and image analysis. Cells $\left(5 \times 10^{7}\right)$ were lysed in urea-thiourea buffer $\{2 \mathrm{M}$ thiourea, $7 \mathrm{M}$ urea, 4\% 3-[(3-cholamidopropyl)-dimethylammonio]-1-propanesulfonate (CHAPS), $1 \mathrm{mM}$ EDTA, $65 \mathrm{mM}$ dithiothreitol (DTT) (Sigma) and $0.1 \mathrm{~g} / 1$ RNase A, $0.1 \mathrm{~g} / 1$ DNase I\} containing complete protease inhibitor cocktail (1:25; Roche Diagnostics, Mannheim, Germany) for $30 \mathrm{~min}$ on ice and centrifuged at 13,000 rpm (Biofuge Fresco centrifuge; Heraeus), at $4^{\circ} \mathrm{C}$ for $1 \mathrm{~h}$ (12). The protein concentration was determined by the Bradford protocol using a Bradford protein assay kit (Bio-Rad, Hercules, CA, USA). The protein samples were stored at $-80^{\circ} \mathrm{C}$ in aliquots until used. 2-DE was performed as described in previous studies (13).

Separation in the first dimension was performed in a PROTEAN isoelectric focusing (IEF) cell (Bio-Rad) with $1000 \mu \mathrm{g}$ of total protein, which was diluted with $350 \mu \mathrm{l}$ rehydration solution (8 M urea, 4\% CHAPS, $20 \mathrm{mM}$ DTT, $0.5 \%$ immobilized $\mathrm{pH}$ gradient buffer and traces of bromo- phenol blue) on 18-cm ReadyStrip IPG strips (pH 3.0-10.0, NL; Amersham Biosciences, Little Chalfont, UK) according to the manufacturer's instructions with active rehydration and final IEF at 10,000 V until 60,000 V.h. Following equilibration for $2 \times 15$ min with an equilibration solution $[50 \mathrm{mM}$ Tris- $\mathrm{HCl}$ (pH 8.8), $6 \mathrm{M}$ urea, $30 \% \mathrm{w} / \mathrm{v}$ glycerol, $2 \% \mathrm{w} / \mathrm{v}$ SDS, $0.3 \%$ DTT and a trace of bromophenol blue] containing DTT and $1.85 \%$ iodoacetamide successively, the second-dimension separation was performed with $12 \%$ SDS-polyacrylamide gel (1 mm gel thick, 20.5-cm height) using Protein II xi 2-D cell (Bio-Rad), with a constant current of $20 \mathrm{~mA} / \mathrm{gel}$ for the initial $40 \mathrm{~min}$ and $30 \mathrm{~mA} / \mathrm{gel}$ thereafter. Gels were stained with colloidal Coomassie Brilliant Blue R-350 (Sigma) and compared with ImageMaster 2D Platinum software (Amersham Biosciences). Samples for each cell line were performed 3 times before determining the final differential spots.

Matrix-assisted laser desorption ionization-time-of-flight (MALDI-TOF)-mass spectrometric analysis and database search. Spots of interest were excised manually and destained with the destaining solution $(15 \mathrm{mM}$ potassium ferricyanide, $50 \mathrm{mM}$ sodium thiosulfate) and then in-gel digestion was performed as previously described $(13,14)$. Samples were cleaved with $1: 1$ ratio by matrix ( $\alpha$-cyano-4-hydroxycinnamic acid in $50 \%$ acetonitrile $/ 0.1 \%$ trifluoroacetic acid) and sample solution. Peptides were separated by high-performance liquid chromatography and analyzed using Bruker Reflex III MALDI-TOF mass spectrometer (Bruker Daltonics, Bremen, Germany). For MALDI peptide mapping, Mascot search engines (www. matrixscience.com) were employed for searching Swiss-Prot (us.expasy.org) and NCBInr databases (www.ncbi.nlm.nih.gov).

Western blot analysis. Protein extracts were prepared from exponentially growing cells by Laemmli sample buffer. Protein concentrations were determined by the Bio-Rad assay (all were from Bio-Rad). For western blot analysis, $60 \mu \mathrm{g}$ of protein from the total cell lysates was fractionated by SDS-polyacrylamide gel electrophoresis (SDS-PAGE). The proteins on these gels were then transferred onto nitrocellulose membranes (Pierce Biotechnology, Inc., Rockford, IL, USA), and the membranes were incubated with the indicated antibodies. After treatment with blocking buffer without 5\% non-fat milk (washing buffer), a dilute solution $(1: 5,000)$ of horseradish peroxidase-linked anti-rabbit goat serum (Cell Signaling Technology, Inc., USA) was added. Membranes were then washed with washing buffer and immune detection was performed using the enhanced chemiluminescence (ECL) kit (Pierce Biotechnology, Inc.). The following primary antibodies and dilutions were used: anti-CFL1, 1:1,000 (Cytoskeleton, Inc., Denver, CO, USA); and anti-p-CFL1, 1:2,000 (a kind gift from Dr James R. Bamburg).

Clinicopathological data. Forty-four patients with ovarian carcinomas were involved in this study. The median age of the patients at diagnosis was 52.6 years (ranging from 39-67 years). The patients involved in this study met the following criteria. i) All patients had primary, histologically proven epithelial ovarian carcinomas. ii) All patients received primary treatment followed by 6-9 cycles of standard chemotherapy, which consisted of taxol $\left(175 \mathrm{mg} / \mathrm{m}^{2}\right)$ combined with cisplatin $\left(75 \mathrm{mg} / \mathrm{m}^{2}\right)$ at 3 -week intervals at the Department 
Table I. Distribution of stage, grade, histological subtypes and patient age in both chemosensitive and taxol-resistant epithelial ovarian carcinoma cases.

\begin{tabular}{|c|c|c|c|c|}
\hline Characteristics & Sensitive $(n=22) n(\%)$ & Resistant $(\mathrm{n}=22) \mathrm{n}(\%)$ & Total $(\mathrm{n}=44) \mathrm{n}(\%)$ & P-value \\
\hline Age (years) & & & & 1.000 \\
\hline$<60$ & $18(81.8)$ & $17(77.3)$ & $35(79.5)$ & \\
\hline$\geq 60$ & $4(18.2)$ & $5(22.7)$ & $9(20.5)$ & \\
\hline Histology & & & & 0.963 \\
\hline Serous papillary cystadenocarcinoma & $12(54.5)$ & $13(59.1)$ & $25(56.8)$ & \\
\hline Mucinous cystadenoma & $2(9.1)$ & $2(9.1)$ & $4 \quad(9.1)$ & \\
\hline Endometriod carcinoma & $5(22.7)$ & $3(13.6)$ & $8(18.2)$ & \\
\hline Transitional cell carcinoma & $1(4.5)$ & $2(9.1)$ & $3(6.8)$ & \\
\hline Clear cell carcinoma & $2(9.1)$ & $2(9.1)$ & $4(9.1)$ & \\
\hline Grade & & & & 1.000 \\
\hline G $1 / 2$ & $5(22.7)$ & $4(18.2)$ & $9(20.5)$ & \\
\hline G 3 & $17(77.3)$ & $18(81.8)$ & $35(79.5)$ & \\
\hline Stage & & & & 0.451 \\
\hline II & $4(18.2)$ & $6(27.3)$ & $10(22.7)$ & \\
\hline III & $17(77.3)$ & $13(59.1)$ & $30(68.2)$ & \\
\hline IV & 1 (4.5) & $3(13.6)$ & $4 \quad(9.1)$ & \\
\hline CA125 (U/ml) & & & & 0.380 \\
\hline$<35$ & 0 & $4(18.2)$ & $4(9.1)$ & \\
\hline$>35$ to $\leq 200$ & $8(36.4)$ & $7(31.8)$ & $15(34.1)$ & \\
\hline$>200$ to $\leq 1,000$ & $7(31.8)$ & $5(22.7)$ & $12(27.3)$ & \\
\hline$>1,000$ to $\leq 3,000$ & $5(22.7)$ & $4(18.2)$ & $9(20.5)$ & \\
\hline$>3,000$ & $2(9.1)$ & $2(9.1)$ & $4(9.1)$ & \\
\hline Chemotherapy & & & & 0.728 \\
\hline Paclitaxel + DDP & $17(77.3)$ & $16(72.7)$ & $33(75.0)$ & \\
\hline $\mathrm{DDP}+\mathrm{CTX}$ & $5(22.7)$ & $6(27.3)$ & $11(25.0)$ & \\
\hline
\end{tabular}

DDP, cisplatin; CTX, cyclophosphamide.

of Gynecology and Obstetrics of the Peking Union Medical College Hospital between 2002 and 2005. iii) The evaluation of response to chemotherapy was based on the information available within an interval of 3-26 months following the beginning of chemotherapy. Chemosensitive patients were defined as demonstrating a complete response to chemotherapy, with a platinum-free interval $>6$ months. Chemoresistant patients were defined as demonstrating a complete response to chemotherapy, following a platinum-free interval $<6$ months; best response to chemotherapy was partial remission, a stable state or progressive disease (15). All cases were staged according to the criteria of the International Federation of Gynecologists and Obstetricians (FIGO). The medical charts of all patients were reviewed and information regarding age at diagnosis, tumor stage, date and type of initial debulking surgery, residual tumor size after initial surgery (defined as the diameter of the largest individual nodule or plaque after initial surgery), date and type of chemotherapy, number of cycles, date of re-cytoreductive surgery, serum CA125 level and status at last follow-up was recorded. Histological types included serous, mucinous, endometrioid, Brenner and clear cell carcinomas. A clinical data summary is presented in Table I. There was no significant difference between chemosensitive and chemoresistant groups in age, FIGO stage and histological subtype. All paraffin-embedded tissue blocks were available for analysis from the primary tumor and/or from the tumor obtained during re-cytoreductive surgery following chemotherapy.

Immunohistochemistry (IHC) techniques. Immunohistochemical studies were performed on paraffin-embedded tumor specimens obtained at the time of initial debulking or re-cytoreductive surgery, using the avidin-biotin complex (ABC) method as previously described $(16,17)$. Briefly, tissue sections were deparaffinized in toluene, rehydrated in graded alcohols and soaked for $5 \mathrm{~min}$ in $3 \%$ hydrogen peroxide to block endogenous peroxidase. After being washed in phosphate-buffered saline (PBS), the slides were incubated with antibody. This was followed by a biotinylated secondary rabbit antibody (Envision + R system) and the ABC complex (Dako, Denmark).

Both positive and negative controls were used. Normal kidney and brain were used as positive controls for CFL1 and p-CFL1, respectively. Negative controls were obtained by replacing primary antibodies with PBS. 
Table II. Cytotoxicity of the two taxol-sensitive and three taxol-resistant sublines.

\begin{tabular}{lcc}
\hline Cell lines & $\mathrm{IC}_{50}(\mu \mathrm{M})$ & $\mathrm{RI}$ (resistant index) \\
\hline SKOV3 & $1.02 \pm 0.35$ & 1 \\
SKOV3/TR2500 & $328.83 \pm 58.60$ & $261.98 \pm 32.89^{\mathrm{b}}$ \\
SKOV3/TR30 & $757.46 \pm 80.85$ & $622.76 \pm 71.37^{\mathrm{b}}$ \\
A2780 & $2.25 \pm 0.69$ & 1 \\
A2780/TR & $21.72 \pm 3.14$ & $8.96 \pm 2.01^{\mathrm{b}}$ \\
\hline
\end{tabular}

${ }^{\mathrm{a}} \mathrm{P}<0.05,{ }^{\mathrm{b}} \mathrm{P}<0.01$.

The immunostaining was interpreted by a professional pathologist without knowledge of the clinical course of the disease. Two features of the immunoreactions were recorded using a semi-quantitative scale: the relative number of positive cells $(0,<25,25-50,>50-75$ and $>75 \%)$ and the intensity of the reaction $(-,+,++,+++,++++)$. The pattern of immunostaining (membrane, cytoplasmic) was also recorded separately. The results were interpreted as negative $\left(0\right.$ and $\left.1^{+}\right)$or positive/overexpressed $\left(2^{+} / 3^{+}\right)$according to the scoring system as previously described (15).

Statistical analysis. Results are expressed as the means \pm SD of more than 3 repeated experiments. Statistical analyses were carried out by One-way ANOVA, Student's t-test and Chi-square test. If necessary, data were logarithmically converted into a normal distribution of variables to remove heterogeneity of variance before analysis. The Kolmogorov-Smirnov test and Mann-Whitney U test were used to evaluate the association of immunostaining with taxol resistance. The proportion of concordant pairs $(\mathrm{P})$ along with its $95 \%$ confidence interval were selected to compare the immunostaining in the taxol-sensitive and -resistant groups. In all tests, $\mathrm{P}<0.05$ was considered to indicate a statistically significant difference. All statistical tests were performed using the SPSS software (v.14.0).

\section{Results}

Characteristics of the taxol-resistant cell lines. Taxol-resistant sublines were established from SKOV3 by episodic exposure to taxol over a period of 16 months. The resultant taxol-resistant sublines were then maintained and passaged in a drug-free medium. The stability of drug resistance was examined at monthly intervals. $\mathrm{IC}_{50}$ and the resistance index (RI) values of the 5 cell lines are shown in Table II and Fig. 1. The resistant phenotype was significantly stable as demonstrated by the $\mathrm{IC}_{50}$ and RI values that had no significant change during a period of 4 months (except SKOV3/TR2500 in 2 months) in a drug-free medium.

Protein screening between the taxol-sensitive and the taxolresistant ovarian cancer cell lines through 2-DE. To discover proteins which have differential expression between the taxol-resistant clones and their parental cell lines, proteomics technology was selected to reveal differences in posttranslational modifications that may be relevant to the protein
A

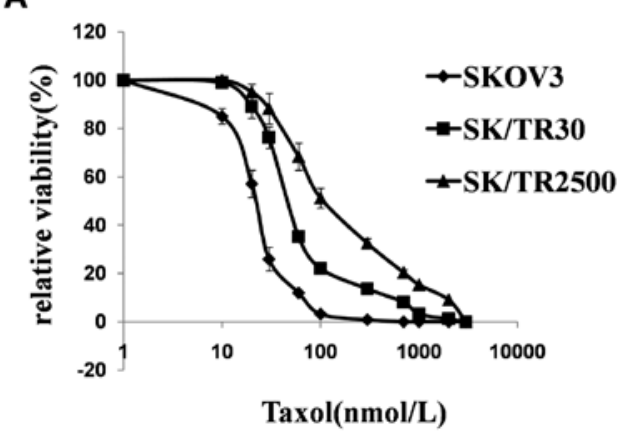

B

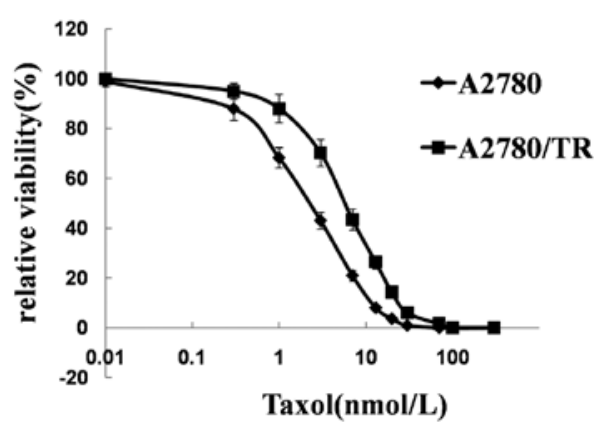

Figure 1. A, Survival curves for the SKOV3 cell line and its derivative taxolresistant sublines as determined by a drug sensitivity assay. B, Survival curves for the A2780 cell line and its derivative taxol-sensitive sublines as determined by a drug sensitivity assay.

function. Total cell extracts of the 2 taxol-sensitive cell lines (SKOV3, A2780) and the 3 taxol-resistant cell lines (SKOV/ TR2500, SKOV/TR30 and A2780/TR) were compared by 2-DE (Fig. 2A). Samples of each cell line were assessed 3 times before determining the final differential spots.

All 1,003 spots were detected on each gel by the autodetect spots analysis software and manual clean-up after Coomassie Brilliant Blue R-350 staining. Approximately 95\% of all spots were matched on duplicate gels and the intensity of the identical spot from different duplicate gels demonstrated no significant change. All the maps showed great similarity between the resistant sublines and their parental cell lines in which the matching rate reached $90 \%$. The matching rate ranged from 80 to $85 \%$ between the 2 different parental cell lines, SKOV3 and A2780. In the matched spots, a 2-fold or higher difference in spot intensity was considered significant. The pI (isoelectric point) of the differentially expressed spots mostly ranged between 5 and 9, and the molecular weight was 14-70 kDa (Tables III and IV). Resistance-dependent differences in expression are highlighted with arrows (Fig. 2A).

Identification of candidate protein spots through mass spectrometry. Twenty-two protein spots in all samples were observed to be significantly different in spot intensity by statistical analysis $(\mathrm{P}<0.05), 16$ of which were identified by matrix-assisted laser desorption ionization-time-of-flight-mass spectrometry (MALDI-TOF-MS) analysis (Tables III and IV). Protein identification was repeated at least once using spots from different gels in order to guarantee reliability. The results showed that the matched spots from different gels 


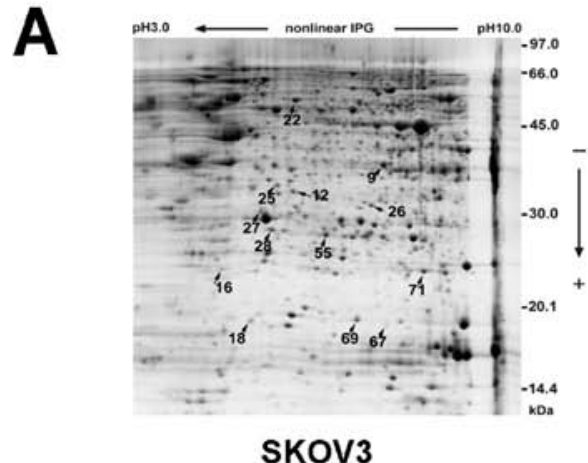

SKOV3

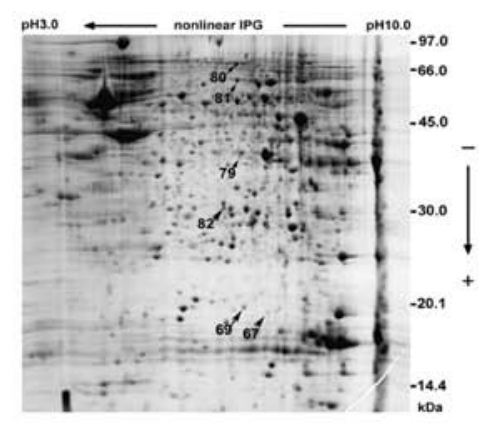

A2780

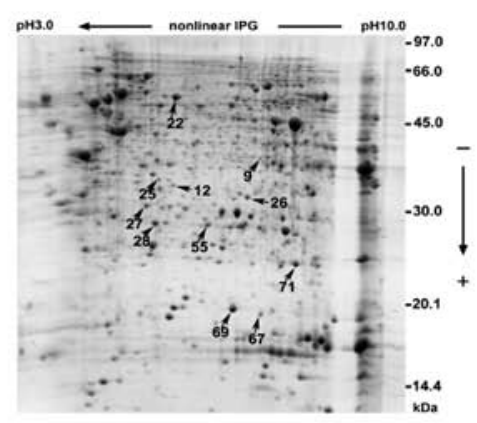

SK/TR30

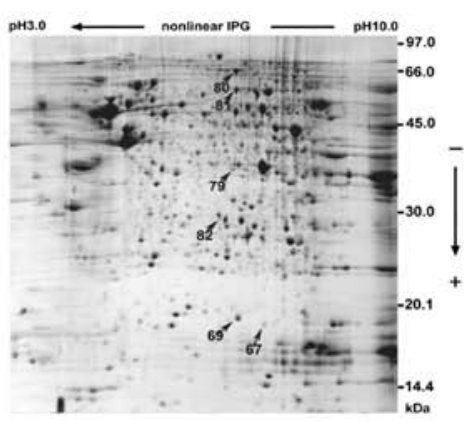

A2780/TR

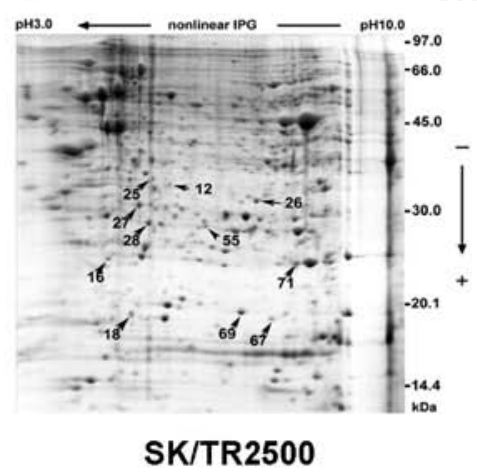

B

1. cofilin 1

2. destrin

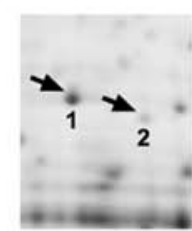

SKOV3

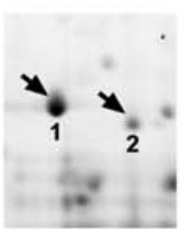

SK/TR30

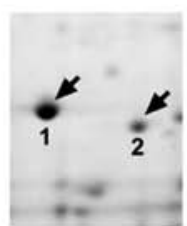

SK/TR2500

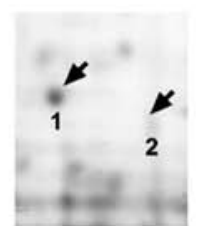

A2780

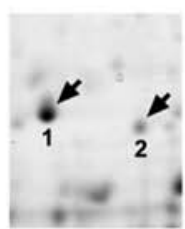

A2780/TR

Figure 2. (A) Representative overview of Coomassie Brilliant Blue-stained 2-DE map of the 2 taxol-sensitive and the 3 taxol-resistant cell lines. All differentially expressed proteins between the taxol-resistant cell lines and their parental cell lines (taxol-sensitive cell lines) are marked with an arrow and numbered on the map. (B) The change in expression of cofilin 1 and destrin coexisted in all 5 cell lines.

were the same protein. A total of 16 proteins were found to be differentially expressed between SKOV3 compared to SKOV3/TR2500, SKOV3 compared to SKOV3/TR30 and A2780 compared to A2780/TR protein preparations. Twelve of the proteins were upregulated and 4 were downregulated in the resistant sublines (Tables III and IV), among which cofilin 1 (CFL1) and destrin were the only 2 spots displaying differences in all 3 taxol-resistant cell lines compared with their parental cell lines (Fig. 2B and Table III). The alteration of CFL1 was the most marked with the upregulated range from 3- to 20-fold and that of destrin was 2- to 10-fold in all of the differentially expressed proteins. The score for protein matching by Mascot for the CFL1 (spot no. 69) was much higher compared to destrin (spot no. 67). These results suggest that CFL1 is a candidate protein which may be correlated with taxol resistance in ovarian cancer cell lines.

Upregulated CFL1 in taxol-resistant cell lines is the phosphorylated form of CFL1. After CFL1 was detected through 2-DE and MALDI-TOF-MS, CFL1 expression in all cell lines was validated through western blot analysis. The result revealed that there was no significant difference in the expression of CFL1 between the taxol-resistant and taxol-sensitive cell lines (Fig. 3).
It is known that CFL1 may be phosphorylated at the serine-3 residue, and this phosphorylated form is an active form which leads to the inhibition of CFL1 as an actin depolymerization factor (16). In addition, dephosphorylated CFL1 and p-CFL1 have a similar molecular weight. Since these forms may not be differentiated by SDS-PAGE, we detected the level of p-CFL1 in vitro. The result showed that the phosphorylated form was overexpressed in taxol-resistant cells compared to the parental ones $(\mathrm{P}<0.01)$ (Fig. 3). These findings indicated that the phosphorylated form of CFL1 was upregulated in taxol-resistant cells but not the total CFL1 in vitro.

Considering that taxol-resistant cell lines were induced in vitro during at least 12 months and surrounding factors may influence the level of CFL1, especially p-CFL1, we detected the levels of p-CFL1 and CFL1 in chemoresistant and chemosensitive cell lines (SKOV3 and SKOV3/TR2500, A2780 and A2780/TR) after being cultured in DMEM/HG without FBS for $48 \mathrm{~h}$ to deplete the effects of the environmental factors (such as FBS). p-CFL1 in taxol-resistant cells remained at a high level while it decreased in taxol-sensitive cells $(\mathrm{P}<0.05)$. When taxol-resistant cell lines were stimulated by taxol, the concentrations of p-CFL1 and CFL1 remained stable and did not display time dependence in our experimental conditions. 
Table III. Upregulated proteins in the 3 resistant sublines identified by MADI-TOF-MS.

\begin{tabular}{|c|c|c|c|c|c|c|c|c|c|}
\hline \multirow[b]{2}{*}{ Spot } & \multirow[b]{2}{*}{ Protein name } & \multirow{2}{*}{$\begin{array}{l}\text { Theoretical } \\
\text { NCBInr ID }^{\mathrm{a}}\end{array}$} & \multirow[b]{2}{*}{$\operatorname{Mr}(\mathrm{Da}) / \mathrm{pI}$} & \multicolumn{2}{|c|}{ Peptides } & \multirow{2}{*}{$\begin{array}{l}\text { Sequence } \\
\text { coverage }\end{array}$} & \multirow[b]{2}{*}{ Score $^{b}$} & \multirow{2}{*}{$\begin{array}{l}\text { Biological } \\
\text { function }\end{array}$} & \multirow[b]{2}{*}{ Cell lines } \\
\hline & & & & Match & Total & & & & \\
\hline 69 & Cofilin 1 & gil5031635 & $18491 / 8.26$ & 7 & 12 & $51 \%$ & 132 & Cytoskeleton & $\begin{array}{l}\text { SK/TR30 } \\
\text { SK/TR2500 } \\
\text { A2780/TR }\end{array}$ \\
\hline 67 & Destrin & gil5802966 & $18493 / 8.51$ & 5 & 11 & $32 \%$ & 65 & Cytoskeleton & $\begin{array}{l}\text { SK/TR30 } \\
\text { SK/TR2500 } \\
\text { A2780/TR }\end{array}$ \\
\hline 01 & Villin2 & gil21614499 & $69199 / 5.94$ & 47 & 69 & $60 \%$ & 130 & Cytoskeleton & A2780/TR \\
\hline 81 & $\begin{array}{l}\text { T-complex protein } 1, \\
\gamma \text { subunit }(\mathrm{TCP}-1)\end{array}$ & gil20455521 & $60364 / 6.46$ & 31 & 43 & $49 \%$ & 196 & Cytoskeleton & A2780/TR \\
\hline 28 & Heat shock protein 27 & gil54696638 & $22768 / 5.98$ & 15 & 57 & $73 \%$ & 148 & Chaperone & $\begin{array}{l}\text { SK/TR30 } \\
\text { SK/TR2500 }\end{array}$ \\
\hline 27 & Prohibitin & gil46360168 & $29802 / 5.57$ & 14 & 46 & $67 \%$ & 140 & Chaperone & $\begin{array}{l}\text { SK/TR30 } \\
\text { SK/TR2500 }\end{array}$ \\
\hline 79 & Lasp-1 & gil5453710 & 29786/6.11 & 22 & 33 & $47 \%$ & 69 & Chaperone & A2780/TR \\
\hline 26 & $\begin{array}{l}\text { Proteasome subunit, } \\
\alpha \text { type } 1\end{array}$ & gil30582133 & $29579 / 6.15$ & 15 & 55 & $42 \%$ & 68 & Catalytic proteins & $\begin{array}{l}\text { SK/TR30 } \\
\text { SK/TR2500 }\end{array}$ \\
\hline 16 & $\begin{array}{l}\text { ATP synthase D chain, } \\
\text { mitochondrial }\end{array}$ & gil23273230 & $18348 / 5.22$ & 10 & 17 & $72 \%$ & 130 & Catalytic activity & SK/TR2500 \\
\hline 71 & $\begin{array}{l}\text { Superoxide dismutase 2, } \\
\text { mitochondrial (SOD2) }\end{array}$ & gil10835187 & $24735 / 8.35$ & 14 & 22 & $68 \%$ & 133 & Redox regulation & $\begin{array}{l}\text { SK/TR30 } \\
\text { SK/TR2500 }\end{array}$ \\
\hline 18 & Stathmin 1 & gil15680064 & $17292 / 5.76$ & 9 & 14 & $51 \%$ & 101 & Signal transduction & SK/TR2500 \\
\hline 22 & $\begin{array}{l}\text { Protein disulfide- } \\
\text { isomerase, } \\
\text { ER60 precursor }\end{array}$ & gil2245365 & $56748 / 5.91$ & 24 & 49 & $53 \%$ & 140 & Metabolic enzyme & SK/TR30 \\
\hline
\end{tabular}

${ }^{\text {aID }}$, identification; ${ }^{\text {ba score of }}>63$ was considered significant $(\mathrm{P}<0.05)$.

Table IV. Downregulated proteins in 3 resistant sublines identified by MADI-TOF-MS.

\begin{tabular}{|c|c|c|c|c|c|c|c|c|c|}
\hline \multirow[b]{2}{*}{ Spot } & \multirow[b]{2}{*}{ Protein name } & \multirow[b]{2}{*}{ NCBInr ID ${ }^{\mathrm{a}}$} & \multirow{2}{*}{$\begin{array}{l}\text { Theoretical } \\
\mathrm{Mr}(\mathrm{Da}) / \mathrm{pI}\end{array}$} & \multicolumn{2}{|c|}{ Peptides } & \multirow{2}{*}{$\begin{array}{l}\text { Sequence } \\
\text { coverage }\end{array}$} & \multirow[b]{2}{*}{ Score $^{b}$} & \multirow{2}{*}{$\begin{array}{l}\text { Biological } \\
\text { function }\end{array}$} & \multirow[b]{2}{*}{ Cell lines } \\
\hline & & & & Match & Total & & & & \\
\hline 25 & Annexin III & gil1421662 & $36222 / 5.63$ & 20 & 36 & $57 \%$ & 157 & Protein transportation & $\begin{array}{l}\text { SK/TR30 } \\
\text { SK/TR2500 }\end{array}$ \\
\hline 12 & Annexin IV & gil1703319 & $35729 / 5.85$ & 21 & 38 & $58 \%$ & 86 & Protein transportation & $\begin{array}{l}\text { SK/TR30 } \\
\text { SK/TR2500 }\end{array}$ \\
\hline 9 & Annexin I & gil404271 & $38559 / 6.64$ & 23 & 34 & $63 \%$ & 199 & Protein transportation & SK/TR30 \\
\hline 55 & Peroxiredoxin 6 & gil4758638 & $24888 / 6.02$ & 14 & 20 & $52 \%$ & 131 & Redox regulation & $\begin{array}{l}\text { SK/TR30 } \\
\text { SK/TR2500 }\end{array}$ \\
\hline
\end{tabular}

aD, identification; b a score of $>63$ was considered significant $(\mathrm{P}<0.05)$.

In taxol-sensitive cell lines, the level of p-CFL1 increased and CFL1 was unchanged; $\beta$-actin was chosen as a reference (Fig. 4). From the above mentioned results, we conclude that p-CFL1 is associated with the drug resistance of taxol in ovarian cancer cell lines, and the surrounding factors did not influence the high level of p-CFL1 in taxol-resistant cells. The higher expression of p-CFL1 may be considered a characteristic of taxol-resistant cells.

Validation of overexpression of p-CFL1 in chemoresistant ovarian cancer tissues by immunostaining. We identified that p-CFL1 was correlated with ovarian cancer cell resistant to 

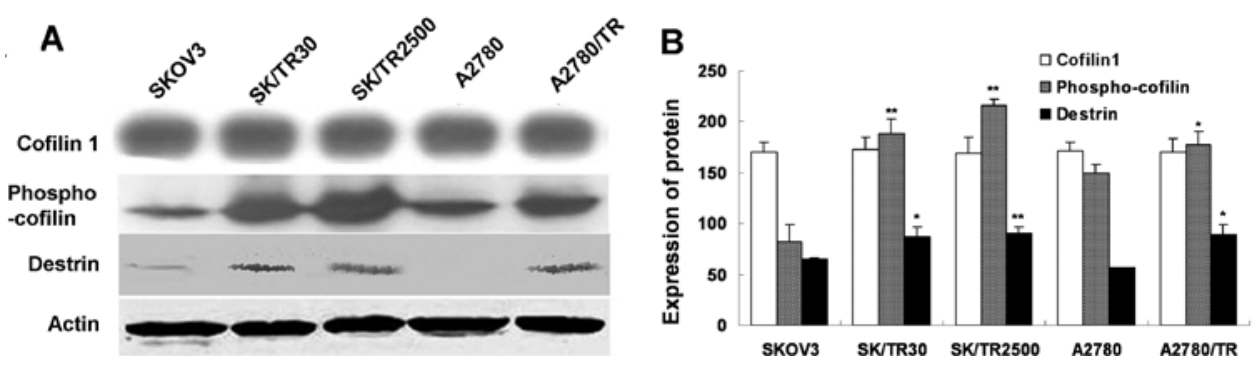

Figure 3. Comparison of different cofilin forms in taxol-sensitive and -resistant ovarian cancer cells. Western blot characterization of CFL1, p-CFL1 and destrin in the 3 resistant and parental cell lines. The level of p-CFL1 and destrin increased significantly in taxol-resistant cells but not CFL1. These results were consistent with 2-DE.

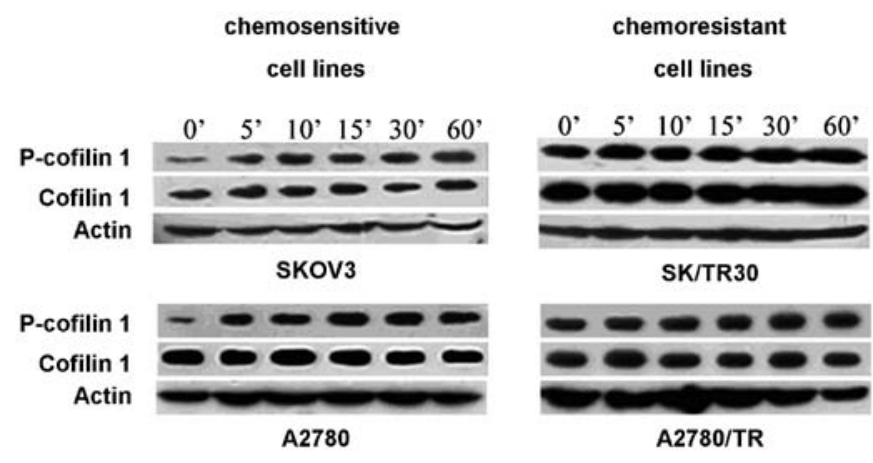

Figure 4. The relationship between taxol treatment and the protein expression of CFL1 and p-CFL1 in taxol-sensitive and taxol-resistant cell lines. The expression levels of p-CFL1 and CFL1 in the 4 cell lines after being stimulated by $10 \mathrm{nM}$ taxol for $0,5,10,15,30,60$ min were identified by western blot analysis. The level of p-CFL1 in the 2 chemoresistant cell lines remained at a high level regardless of whether or not they were stimulated with taxol while the level of p-CFL1 varied in chemosensitive cell lines. Actin was used as the internal standard.

taxol in vitro. Furthermore, we also detected the expression levels of CFL1 and p-CFL1 in primary human ovarian cancer tissues.

Immunostaining of CFL1 was positive in $86.4 \%(19 / 22)$ of the chemosensitive and $90.9 \%(20 / 22)$ of the chemoresistant ovarian carcinomas, but this was not statistically significant ( $\mathrm{P}=0.991, \mathrm{U}=263.6$ ) (Fig. 5a, b and f). However, immunostaining of p-CFL1 was positive in $77.3 \%(17 / 22)$ of chemosensitive and in $95.9 \%(21 / 22)$ of the chemoresistant ovarian carcinomas, which was statistically significant $(\mathrm{P}=0.014<0.05, \mathrm{U}=157.5)$ (Fig. 5c-e and $\mathrm{g})$. These results indicated that $\mathrm{p}-\mathrm{CFL} 1$ in taxol-resistant ovarian cancer tissues was overexpressed in vivo and in vitro.

\section{Discussion}

The goal of this study was to detect proteins which may be correlated with chemoresistance to taxol in ovarian cancer, which may assist us in the early diagnosis of taxol chemoresistance in clinical treatment. Although the results of the experiments with cells in culture may not always mirror the situation in human tumors in vivo, we employed 2 schemes for assessing toxicity using one cell line (SKOV3), together with other taxol-sensitive and -resistant cell lines (A2780 and A2780/TR) and proteomic analysis to report markers of taxol resistance.
2-DE analysis comparing the taxol-resistant with the sensitive cell lines yielded several differentially expressed proteins. Twelve proteins were identified by mass spectrometry to have upregulated expression: CFL1, destrin, villin2, TCP-1, hsp27, prohibitin, lasp-1, proteasome subunit- $\alpha$ type, SOD2, stathmin 1, and ER60 precursor, and 4 proteins were downregulated: Annexin 3, Annexin 4, Annexin 1 and peroxiredoxin 6. Approximately half of the differentially expressed proteins identified in this study are related to the organization or activity of the actin cytoskeleton, which may explain the failure of strategies based on changes in the expression of P-glycoprotein and tubulin in clinical treatment. Although alterations of actin associated with drug resistance have been reported, hsp27 and stathmin were also confirmed to be relevant to drug resistance in previous studies (18-21). Unfortunately, their high expression was not observed in these resistant cell lines. Only 2 protein spots, CFL1 and destrin, were upregulated in all 3 taxol-resistant cell lines, which were unexpected candidates for drug resistance. CFL1 and destrin are both actin-depolymerizing proteins and are involved in the organization of the cytoskeleton. A number of authors have reported CFL1 and destrin differential expression in cancer cells $(13,22-25)$. To the best of our knowledge, we report for the first time their function related with drug resistance in ovarian cancer. Since the score of CFL1 (spot no. 69) for protein matching by Mascot was much higher than destrin (spot no. 67), as well as destrin and CFL1 sharing $70 \%$ identical sequence of DNA (26-27), the study was focused on CFL1. However, the results of the western blot analysis showed that the expression levels of CFL1 in all cell lines were similar. It is known that CFL1 has phosphorylated and dephosphorylated forms, and these forms may be differentiated from each other by their different isoelectric points (27). Based on the above-mentioned fact, we detected the level of p-CFL1 and found a high level of p-CFL1 in the taxol-resistant cell lines. As a result, the differential protein spot in 2-D gel was possibly p-CFL1 not CFL1.

CFL1 is one of the major proteins responsible for cell migration processes, playing a key role in actin filament dynamics and apoptosis induced by oxidants (28-30). Bernstein and Bamburg suggest that CFL1 plays a major role in cell biology, and that any interference with its normal activity is likely to have severe repercussions (29). The spontaneous overexpression of CFL1 may be detected in invasive sub-populations of breast tumor cells in rats, as well as in biopsies of oral, renal, non-small cell lung cancer (NSCLC) and ovarian carcinoma. 

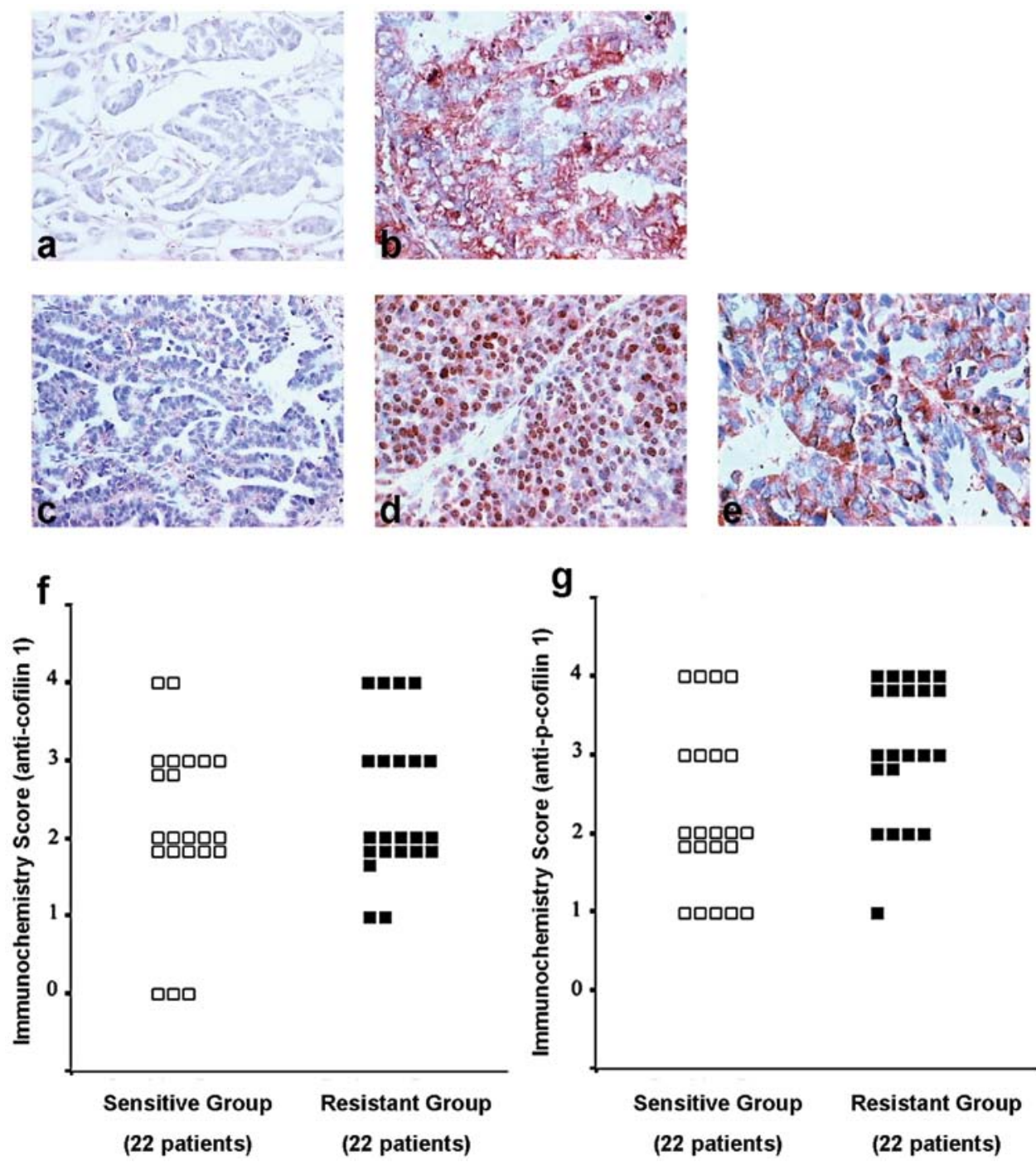

Figure 5. Results of immunostaining of CFL1 and phospho-cofilin 1 in primary ovarian cancer tissues. Immunostaining of CFL1: (a) negative; and (b) positive staining in the cytoplasm. Immunostaining of phospho-cofilin 1: (c) negative and (d) positive staining in the nucleus, (e) positive staining in the cytoplasm. (f) Scores for CFL1 and p-CFL1 immunohistochemical staining in ovarian cancer sections. Tumor sections were obtained from 22 chemosensitive and 22 chemoresistant patients. Analysis with Mann-Whitney U test shows that there is no significant difference between the 2 groups, (g) scores of p-CFL1 immunohistochemical staining in ovarian cancer sections. Analysis with Mann-Whitney U test shows that there is a significant difference between the 2 groups.

High CFL1 levels are correlated with lower overall survival rates and resistance to several alkylating drugs in NSCLC patients (13,23-25). The overexpression of CFL was also detected in cisplatin-resistant cells (13). However, there are no reports demonstrating what role CFL plays in drug resistance. Our research suggests that the phosphorylation of CFL1 was involved in taxol-resistance. In our study, CFL1 was selected through proteomic analysis and we investigated its relationship with taxol resistance. After in vitro and in vivo identification of the results of 2-DE, we found that the level of CFL1 did not increase whereas p-CFL1 increased significantly. CFL1 has been known to promote actin depolymerization and filament severing. Several experiments suggested that this feature of CFL1 was involved in its inhibitory action. Both its actin depolymerization activity and its inhibitory action on the receptor were dependent on its phosphorylation state (31). A strong differential expression of p-CFL1 between taxol-sensitive and -resistant cell lines in the proteomic analysis suggested that an alteration in the cellular levels of p-CFL1 may be associated with drug resistance. Considering that the chemoresistant cells were induced in vitro for approximately 1 year, we removed the effects of the surrounding factors as far as possible and identified that the level of p-CFL1 in taxol-resistant cells was not effected by the surrounding factors (such as FBS). We also discovered that p-CFL1 could stably exist at high levels in taxol-resistant cells which may be used as a marker of taxol-resistant cells. Fortunately in the clinical sample, similar results were obtained. Thus, we speculated that high levels of p-CFL1 expression may be associated with epithelial ovarian cancer cell resistance to chemotherapy in vitro.

A number of researchers (31) have identified that the activity of CFL1 is reversibly regulated by phosphorylation and dephosphorylation, with the dephosphorylated form being inactive. Based on our results, we suggested that CFL1 functions in chemoresistance and yet must first pass through the process of phosphorylation. LIM-kinase (LIMK) and TES-kinase are possibly responsible for this site phosphorylation and thereby inactivate CFL1 $(31,32)$. It is necessary to 
fully elucidate the pathways by which the dephosphorylated CFL1 or p-CFL1 affect drug resistance.

In conclusion, our study indicates the potency of a proteomic approach to study drug resistance in cancer cells. These findings support that p-CFL1 may be an important regulator in the development of taxol resistance. Clearly, a greater number of investigations are required to elucidate how the protein acts in the taxol activity pathway that may induce taxol resistance in epithelial ovarian cancer cells. Its distinct function in the regulation of taxol resistance encourages us to pursue the use of marker proteins as a clinical utility for early detection of drug resistance and for preventing a poor prognosis.

\section{Acknowledgements}

We thank Dr James R. Bamburg (University of California, San Francisco, USA) for the kind gift of anti-p-CFL1, Dr Theo Rein (Max Planck Institute for Psychiatry, Munich, Germany) for the kind gift of plasmid pRK5cof, Dr Sutherland K. Maciver (University of Edinburgh, Scotland, UK) for the kind gift of pCFL-EGFP and Dr Xuemin Zhang and colleagues (Institute of Basic Medical Sciences, National Center of Biomedical Analysis, Beijing, China) for the proteomic technical assistance. We again thank Dr James R. Bamburg and Dr Sutherland K. Maciver for critical comments regarding the manuscript and helpful discussions. This study was supported by the Key Research Foundation of Peking Union Medical College Hospital (200203*).

\section{References}

1. Cicchillitti L, Della Corte A, Di Michele M, Donati MB, Rotilio D and Scambia G: Characterisation of a multimeric protein complex associated with ERp57 within the nucleus in paclitaxel-sensitive and -resistant epithelial ovarian cancer cells: The involvement of specific conformational states of $\beta$-actin. Int J Oncol 37: 445-454, 2010.

2. Bovicelli A, D'Andrilli G and Giordano A: New players in ovarian cancer. J Cell Physiol 226: 2500-2504, 2011.

3. Kurman RJ and Shih IeM: The origin and pathogenesis of epithelial ovarian cancer: a proposed unifying theory. Am J Surg Pathol 34: 433-443, 2010.

4. Kim A, Ueda Y, Naka T and Enomoto T: Therapeutic strategies in epithelial ovarian cancer. J Exp Clin Cancer Res 31: 14, 2012.

5. Rein DT, Volkmer A, Bauerschmitz G, et al: Combination of a MDR1-targeted replicative adenovirus and chemotherapy for the therapy of pretreated ovarian cancer. J Cancer Res Clin Oncol 138: 603-610, 2012.

6. Kavallaris M: Microtubules and resistance to tubulin-binding agents. Nat Rev Cancer 10: 194-204, 2010.

7. Umezu T, Shibata K, Kajiyama H, et al: Taxol resistance among the different histological subtypes of ovarian cancer may be associated with the expression of class III beta-tubulin. Int J Gynecol Pathol 27: 207-212, 2008.

8. Wang Y, Chen Q, Jin S, et al: Up-regulation of P-glycoprotein is involved in the increased paclitaxel resistance in human esophageal cancer radioresistant cells. Scand J Gastroenterol 47: 802-808, 2012

9. Yan XD, Li M, Yuan Y, Mao N and Pan LY: Biological comparison of ovarian cancer resistant cell lines to cisplatin and Taxol by two different administrations. Oncol Rep 17: 1163-1169, 2007.

10. Carmichael J, DeGraff WG, Gazdar AF, Minna JD and Mitchell JB: Evaluation of a tetrazolium-based semiautomated colorimetric assay: assessment of chemosensitivity testing. Cancer Res 47: 936-942, 1987.

11. Preusser M, Spiegl-Kreinecker S, Lötsch D, et al: Trabectedin has promising antineoplastic activity in high-grade meningioma. Cancer: March 5, 2012 (Epub ahead of print). doi: 10.1002/ cncr. 27460
12. Gorg A, Obermaier C, Boguth G, et al: The current state of twodimensional electrophoresis with immobilized $\mathrm{pH}$ gradients. Electrophoresis 21: 1037-1053, 2000.

13. Yan XD, Pan LY, Yuan Y, Lang JH and Mao N: Identification of platinum-resistance associated proteins through proteomic analysis of human ovarian cancer cells and their platinumresistant sublines. J Proteome Res 6: 772-780, 2007.

14. Joubert-Caron R, Le Caer JP, Montandon F, et al: Protein analysis by mass spectrometry and sequence database searching: a proteomic approach to identify human lymphoblastoid cell line proteins. Electrophoresis 21: 2566-2575, 2000.

15. Swenerton K, Muss H and Robinson E: Salvage Chemotherapy for Refractory Disease. In: Ovarian Cancer Controversies on Management. Gershenson DM, McGuire WP (eds). Churchill Livingstone, New York, pp169-194, 1998.

16. Hsu SM, Raine L and Fanger H: Use of avidin-biotin-peroxidase complex $(\mathrm{ABC})$ in immunoperoxidase techniques: a comparison between $\mathrm{ABC}$ and unlabeled antibody (PAP) procedures. J Histochem Cytochem 29: 577-580, 1981.

17. Beamer LC, Grant ML, Espenschied CR, et al: Reflex immunohistochemistry and microsatellite instability testing of colorectal tumors for Lynch syndrome among US cancer programs and follow-up of abnormal results. J Clin Oncol 30: 1058-1063, 2012.

18. Tanaka Y, Fujiwara K, Tanaka H, Maehata K and Kohno I: Paclitaxel inhibits expression of heat shock protein 27 in ovarian and uterine cancer cells. Int J Gynecol Cancer 14: 616-620, 2004.

19. Alli E, Yang JM, Ford JM and Hait WN: Reversal of stathminmediated resistance to paclitaxel and vinblastine in human breast carcinoma cells. Mol Pharmacol 71: 1233-1240, 2007.

20. Song TF, Zhang ZF, Liu L, Yang T, Jiang J and Li P: Small interfering RNA-mediated silencing of heat shock protein 27 (HSP27) Increases chemosensitivity to paclitaxel by increasing production of reactive oxygen species in human ovarian cancer cells (HO8910). J Int Med Res 37: 1375-1388, 2009.

21. Balasubramani M, Nakao C, Uechi GT, et al: Characterization and detection of cellular and proteomic alterations in stable stathmin-overexpressing, taxol-resistant BT549 breast cancer cells using offgel IEF/PAGE difference gel electrophoresis. Mutat Res 722: 154-164, 2011.

22. Estornes Y, Gay F, Gevrey JC, et al: Differential involvement of destrin and cofilin-1 in the control of invasive properties of Isrecol human colon cancer cells. Int J Cancer 121: 2162-2171, 2007.

23. Castro MA, Dal-Pizzol F, Zdanov S, et al: CFL1 expression levels as a prognostic and drug resistance marker in nonsmall cell lung cancer. Cancer 116: 3645-3655, 2010.

24. Muller CB, de Barros RL, Castro MA, et al: Validation of cofilin-1 as a biomarker in non-small cell lung cancer: application of quantitative method in a retrospective cohort. J Cancer Res Clin Oncol 137: 1309-1316, 2011.

25. Nishimura S, Tsuda H, Kataoka F, et al: Overexpression of cofilin 1 can predict progression-free survival in patients with epithelial ovarian cancer receiving standard therapy. Hum Pathol 42: 516-521, 2011.

26. Vartiainen MK, Mustonen T, Mattila PK, et al: The three mouse actin-depolymerizing factor/cofilins evolved to fulfill cell-typespecific requirements for actin dynamics. Mol Biol Cell 13: 183-194, 2002.

27. Dai YP,Bongalon S, Mutafova-Yambolieva VN and Yamboliev IA: Distinct effects of contraction agonists on the phosphorylation state of cofilin in pulmonary artery smooth muscle. Adv Pharmacol Sci 2008: 362741, 2008.

28. Klamt F, Zdanov S, Levine RL, et al: Oxidant-induced apoptosis is mediated by oxidation of the actin-regulatory protein cofilin. Nat Cell Biol 11: 1241-1246, 2009.

29. Bernstein BW and Bamburg JR: ADF/cofilin: a functional node in cell biology. Trends Cell Biol 20: 187-195, 2010.

30. Teng B, Lukasz A and Schiffer M: The ADF/cofilin-pathway and actin dynamics in podocyte injury. Int J Cell Biol 2012: 320531, 2012.

31. Ruegg J, Holsboer F, Turck C and Rein T: Cofilin 1 is revealed as an inhibitor of glucocorticoid receptor by analysis of hormoneresistant cells. Mol Cell Biol 24: 9371-9382, 2004.

32. Scott RW, Hooper S, Crighton D, et al: LIM kinases are required for invasive path generation by tumor and tumor-associated stromal cells. J Cell Biol 191: 169-185, 2010. 